\title{
On the Question of an Ultraviolet Zero of the Beta Function of the $\lambda\left(\vec{\phi}^{2}\right)_{4}^{2}$ Theory
}

\author{
Robert Shrock \\ C. N. Yang Institute for Theoretical Physics \\ Stony Brook University \\ Stony Brook, NY 11794
}

\begin{abstract}
We investigate the possibility of an ultraviolet (UV) zero in the $n$-loop beta function of a $\lambda\left(\vec{\phi}^{2}\right)^{2}$ field theory with an $N$-component scalar field, $\vec{\phi}$, in four spacetime dimensions, up to the level of $n=5$ loops. Although the two-loop beta function has a UV zero, we find that the $n$-loop beta function for $n=3,4,5$ either does not have a UV zero or does not have one at a value of $\lambda$ in approximate agreement with the two-loop calculation. Similar results are obtained after application of scheme transformations to the beta function and via calculation of Padé approximants. We thus conclude that in the range of $\lambda$ where the perturbative calculation of the $n$-loop beta function is reliable, the theory does not exhibit robust evidence of a UV zero up to the level of $n=5$ loops.
\end{abstract}

PACS numbers: 11.10.-z,,11.10.Hi

\section{INTRODUCTION}

A subject of fundamental interest in quantum field theory is the dependence of the interaction coupling on the Euclidean momentum scale, $\mu$, where it is measured. This dependence is described by the beta function of the theory [1]. Here we investigate this for a theory with a real, $N$-component, scalar field, $\vec{\phi}=\left(\phi_{1}, \ldots, \phi_{N}\right)^{T}$ with a self-interaction of the form $\lambda\left(\vec{\phi}^{2}\right)^{2}$, in $d=4$ spacetime dimensions (at zero temperature), focusing on the question of whether the beta function exhibits robust evidence of an ultraviolet zero. We study this up to the highest loop order for which the beta function has been calculated, namely five loops. This theory is defined by the path integral

$$
Z=\int \prod_{x}[d \vec{\phi}(x)] e^{i S}
$$

where $S=\int d^{4} x \mathcal{L}$, with the Lagrangian $[2]$

$$
\mathcal{L}=\frac{1}{2}\left(\partial_{\mu} \vec{\phi}\right) \cdot\left(\partial^{\mu} \vec{\phi}\right)-\frac{m^{2}}{2} \vec{\phi}^{2}-\frac{\lambda}{4 !}\left(\vec{\phi}^{2}\right)^{2} .
$$

This theory will be denoted $\lambda\left(\vec{\phi}^{2}\right)_{4}^{2}=\lambda|\vec{\phi}|_{4}^{4}$ for short, where the subscript means $d=4$. The Lagrangian $\mathcal{L}$ is invariant under global $\mathrm{O}(N)$ transformations of the field $\vec{\phi}$. Quantum loop corrections lead to a scale dependence in the physical, renormalized coupling, $\lambda(\mu)$.

The variation of $\lambda(\mu)$ as a function of $\mu$ is described by the beta function

$$
\beta_{\lambda}=\frac{d \lambda}{d t}
$$

where $d t=d \ln \mu$. (The argument $\mu$ will often be suppressed in the notation.) As is well-known, the lowestorder (one-loop) term in this beta function has a positive coefficient, so that as $\mu \rightarrow 0$, the coupling $\lambda(\mu) \rightarrow 0$, i.e., the theory is infrared-free. This perturbative result has been confirmed by nonperturbative approaches [3] and is sometimes referred to as the "triviality" property of the theory. One then interprets the theory as an effective one, applicable over some range of momenta $\mu$ (see, e.g., [4, 5]). Since the one-loop term in $\beta_{\lambda}$ is positive, it follows that as $\mu$ increases from 0 , the coupling $\lambda(\mu)$ also increases. If one were naively to consider only the lowest-order term in the beta function and integrate the differential equation (1.3), then this would lead to a pole in $\lambda(\mu)$ at a finite value of $\mu$. Of course, one would not actually be justified in drawing such an inference, since as $\mu$ increased, $\lambda(\mu)$ would become too large for the perturbative calculation to be valid before the position of the pole would be reached. However, this motivates one to consider higher-loop terms in the beta function.

An important question is whether $\beta_{\lambda}$ has a UV zero, which could thus constitute an ultraviolet fixed point (UVFP) of the renormalization group (RG), so that as $\mu$ increases from the infrared (IR) limit $\mu=0$ to the UV limit $\mu \rightarrow \infty, \lambda(\mu)$ would increase, but approach a finite value. In this paper we shall investigate this question using higher-loop calculations of the beta function up to five-loop order. The two-loop term in $\beta_{\lambda}$ is negative, so that, at the two-loop level, $\beta_{\lambda}$ does, in fact, exhibit a UV zero. If the existence of this zero were to be confirmed at higher loop order, then it would mean that the growth of $\lambda(\mu)$ would be cut off for large $\mu$. Although the $\lambda|\vec{\phi}|_{4}^{4}$ theory has been studied for many years, we are not aware of a paper in the literature that has addressed and answered the question of whether the higher-loop beta function also exhibits robust evidence for a UV zero. A prerequisite for a UV zero of $\beta_{\lambda}$ to be well-established, at least within the context of a perturbative calculation, is that for a given value of $N$, when one calculates the value of the zero to $n$-loop order and to $(n+1)$-loop order, there should not be a large fractional shift in the result. We shall investigate this question both for a range of finite values of $N$ and in the large- $N$ limit. Early discussions of the large- $N$ limit of the $\mathrm{O}(N) \lambda|\vec{\phi}|_{4}^{4}$ theory include [6]; see also [5]. To state our conclusion at the outset, we find evidence against a UV zero in this theory. This finding is 
consistent with the view of this theory as an effective field theory, to be applied only over a restricted range of momentum scales $\mu$. What it contributes to this consensus is a quantitative analysis of the perturbatively calculated $n$-loop beta function up to the rather high order of five loops.

For our study of the ultraviolet properties of the beta function for large momentum scale $\mu$, we take the quartic coupling $\lambda$ in $\mathcal{L}$ to be positive for the stability of the theory. Corrections to the scalar potential have been discussed, e.g., in [7]. We take the coefficient, $m^{2}$, of the term $(1 / 2) \vec{\phi}^{2}$ in $\mathcal{L}$ to be fixed and recall that its value does not enter in the beta function $\beta_{\lambda}$ that we analyze. If one were to consider this scalar theory as being embedded in a larger theory with higher physical mass scales, then one would have to deal with the hierarchy problem, namely the sensitivity, via loop corrections, of $m^{2}$ to these higher mass scales. To focus on the question of a UV zero of the beta function, which is of interest in its own right, we thus consider this theory in isolation.

The question of the existence of a UV zero in the beta function of the $\mathrm{O}(N) \lambda|\vec{\phi}|_{4}^{4}$ is somewhat similar to the question of the existence of a UV zero in the beta function of a $\mathrm{U}(1)$ gauge theory in $d=4$ dimensions with a set of $N_{f}$ fermions of a given charge. Both of these theories are IR-free. In [8] we recently investigated the question for the $\mathrm{U}(1)$ gauge theory up to the five loop level for general $N_{f}$ and in the limit of large $N_{f}$ and concluded that it does not exhibit a UV zero [8] (see also [9]).

An example of a theory with a beta function that has a UV zero is the nonlinear $\sigma$ model $(\mathrm{NL} \sigma \mathrm{M})$ in $d=2+\epsilon$ spacetime dimensions, where one finds, from an exact solution of this model in the limit $N \rightarrow \infty$ (involving a sum of an infinite number of Feynman diagrams that dominate in this limit) the result (for small $\epsilon$ ) [10]

$$
\beta_{\lambda}=\epsilon \lambda\left(1-\frac{\lambda}{\lambda_{c}}\right)
$$

where $\lambda$ is an effective dimensionless coupling in the model and

$$
\lambda_{c}=\frac{2 \pi \epsilon}{N} .
$$

In addition to being an IR-free theory with a UVFP, this was also an early example of a theory which, by perturbative power-counting for $\epsilon>0$, is nonrenormalizable, but nevertheless yields calculable, well-defined predictions via the use of a nonperturbative method, namely the large- $N$ limit. This possibility has been termed "asymptotic safety" [4, 11].

This paper is organized as follows. In Sect. II we analyze the behavior of the $n$-loop coefficients of the beta function of the $\lambda|\vec{\phi}|_{4}^{4}$ theory. In Sect. III we investigate the question of the presence or absence of a UV zero of the beta function up to five-loop order. Sect. IV contains a discussion of the large- $N$ limit. In Sect. V we study the effect of applying scheme transformations to the beta function in the analysis of a possible UV zero. Sect. VI contains a corresponding study using Padé approximants. In Sect. VII we compare our findings with those for some related theories. Our conclusions are summarized in Sect. VIII. Some relevant formulas for discriminants are given in the Appendix. Although we restrict our study here to the beta function of the $\mathrm{O}(N) \lambda|\vec{\phi}|^{4}$ theory in $d=4$ dimensions, we note parenthetically that this field theory has been used extensively to study critical phenomena in $d=4-\epsilon^{\prime}$ dimensions, with particular application to $d=3$, and there has also been interest in the $\lambda\left(\vec{\phi}^{2}\right)^{3}$ theory in $d=3$ dimensions for the study of tricritical points.

\section{BETA FUNCTION AND PROPERTIES OF COEFFICIENTS UP TO FIVE LOOPS}

\section{A. General}

The beta function $\beta_{\lambda}$ of Eq. (1.3) has the series expansion

$$
\beta_{\lambda}=\lambda \sum_{\ell=1}^{\infty} b_{\ell} a^{\ell},
$$

where

$$
a \equiv \frac{\lambda}{16 \pi^{2}} .
$$

An equivalent beta function is $\beta_{a}=d a / d t$, with the series expansion

$$
\beta_{a}=a \sum_{\ell=1}^{\infty} b_{\ell} a^{\ell}
$$

The $n$-loop $\beta_{\lambda}$ and $\beta_{a}$ functions, denoted $\beta_{\lambda, n \ell}$ and $\beta_{a, n \ell}$, are given by Eqs. (2.1) and (2.3) with the upper limit of the loop summation index $\ell=n$ instead of $\ell=\infty$. It will be convenient to define the scaled coefficients

$$
\bar{b}_{\ell} \equiv \frac{b_{\ell}}{(4 \pi)^{\ell}}
$$

for tables to be presented later. In our analysis of how the inclusion of higher loops changes the value of the beta function for a given $a=a(\mu)$ and $N$, it is useful to define the ratio

$$
R_{a, n} \equiv \frac{\beta_{a, n \ell}}{\beta_{a, 1 \ell}}=1+\sum_{\ell=2}^{n}\left(\frac{b_{\ell}}{b_{1}}\right) a^{\ell-1} .
$$

\section{B. $b_{1}$ and $b_{2}$}

The one-loop and two-loop coefficients in the beta function, $b_{1}$ and $b_{2}$, are independent of the scheme used 
for regularization and renormalization, while the coefficients at loop order three and higher, $b_{\ell}$ for $\ell \geq 3$, are scheme-dependent. The first two coefficients are 12 ]

$$
b_{1}=\frac{1}{3}(N+8)
$$

and

$$
b_{2}=-\frac{1}{3}(3 N+14)
$$

As noted above, since $b_{1}>0$, it follows that for small $a$, where the calculation of $\beta_{a}$ is most reliable, $\beta_{a}>0$, so that as $\mu \rightarrow 0, a(\mu) \rightarrow 0$, i.e., the theory is IR-free. Going in the opposite direction, to large $\mu$ in the ultraviolet, $a(\mu)$ increases. A basic question is whether this growth is cut off by a UV zero of the beta function, so that as $\mu \rightarrow \infty, a(\mu)$ approaches a fixed finite constant, $a_{U V}=\lambda_{U V} /\left(16 \pi^{2}\right)$ or whether, in contrast, $\beta_{a}$ has no (reliably calculable) UV zero, so that $a(\mu)$ continues to increase with increasing $\mu$ until it exceeds the regime where the function $\beta_{a}$ describing its evolution can be reliably calculated. As is evident from Eq. 2.7 $b_{2}$ is negative, so the two-loop beta function does have an UV zero. However, one must study whether this is stable when higher-loop terms are included in the beta function. In order to carry out this analysis, we first characterize the behavior of the higher-loop coefficients to the highest order for which they have been calculated, namely $n=5$.

$$
\text { C. } b_{3}
$$

The convenient and widely used $\overline{M S}$ scheme employs dimensional regularization [13] with modified minimal subtraction 14]. In the $\overline{\mathrm{MS}}$ scheme, the three-loop coefficient is [12, 15]

$$
b_{3}=\frac{11}{72} N^{2}+\left(\frac{461}{108}+\frac{20 \zeta(3)}{9}\right) N+\frac{370}{27}+\frac{88 \zeta(3)}{9} .
$$

(See also [16] for a review and [17] for the $N=1$ special case of $b_{3}$ in this scheme). Numerically,

$$
b_{3}=0.15278 N^{2}+6.93976 N+24.4571 .
$$

Here and below, numerical quantities are listed to the indicated floating-point accuracies. For all physical $N$, this coefficient is positive and is a monotonically increasing function of $N$. For later reference, we list values of this and the other coefficients of the beta function, expressed in terms of the conveniently rescaled quantities $\bar{b}_{\ell}$ defined in Eq. (2.4), in Table 1 .

\section{D. $b_{4}$}

The four-loop coefficient in $\beta$, calculated in the $\overline{\mathrm{MS}}$ scheme, is [15]

$$
\begin{aligned}
b_{4} & =\frac{5}{3888} N^{3}+\left(-\frac{395}{243}-\frac{14 \zeta(3)}{9}+\frac{10 \zeta(4)}{27}-\frac{80 \zeta(5)}{81}\right) N^{2}+\left(-\frac{10057}{486}-\frac{1528 \zeta(3)}{81}+\frac{124 \zeta(4)}{27}-\frac{2200 \zeta(5)}{81}\right) N \\
& -\frac{24581}{486}-\frac{4664 \zeta(3)}{81}+\frac{352 \zeta(4)}{27}-\frac{2480 \zeta(5)}{27}
\end{aligned}
$$

Numerically,

$$
\begin{aligned}
b_{4} & =\left(1.2860 \times 10^{-3}\right) N^{3}-4.11865 N^{2}-66.5621 N \\
& -200.92637 .
\end{aligned}
$$

In contrast to the lower-order coefficients $b_{\ell}$ with $\ell=$ $1,2,3, b_{4}$ is neither a monotonic function of $N$ nor of fixed sign. At $N=1, b_{4}=-271.606$ (equivalently, $\bar{b}_{4}=$ -0.010892 ), and as $N$ increases, $b_{4}$ decreases through negative values. This coefficient reaches a minimum value (i.e., $-b_{4}$ reaches a maximum value) at the large number [18] $N=2143.16$ and then increases, passing through zero and becoming positive as $N$ increases through the value $N=N_{b 4 z}$, where

$$
N_{b 4 z}=3218.755
$$

This is the relevant one among the three roots of the cubic equation $b_{4}=0$ (the other two roots occur at negative, and hence unphysical, values of $N$ ). Values of $\bar{b}_{4}$ are given in Table $\amalg$.

\section{E. $b_{5}$}

In the $\overline{\mathrm{MS}}$ scheme the five-loop coefficient is [15]

$$
b_{5}=\left(\frac{13}{62208}-\frac{\zeta(3)}{432}\right) N^{4}+\left(\frac{6289}{31104}+\frac{26 \zeta(3)}{81}-\frac{2 \zeta(3)^{2}}{27}-\frac{7 \zeta(4)}{24}+\frac{305 \zeta(5)}{243}-\frac{25 \zeta(6)}{81}\right) N^{3}
$$




$$
\begin{aligned}
& +\left(\frac{50531}{3888}+\frac{8455 \zeta(3)}{486}-\frac{59 \zeta(3)^{2}}{81}-\frac{347 \zeta(4)}{54}+\frac{7466 \zeta(5)}{243}-\frac{1775 \zeta(6)}{243}+\frac{686 \zeta(7)}{27}\right) N^{2} \\
& +\left(\frac{103849}{972}+\frac{69035 \zeta(3)}{486}+\frac{446 \zeta(3)^{2}}{81}-\frac{2383 \zeta(4)}{54}+\frac{66986 \zeta(5)}{243}-\frac{7825 \zeta(6)}{81}+343 \zeta(7)\right) N \\
& +\frac{17158}{81}+\frac{27382 \zeta(3)}{81}+\frac{1088 \zeta(3)^{2}}{27}-\frac{880 \zeta(4)}{9}+\frac{55028 \zeta(5)}{81}-\frac{6200 \zeta(6)}{27}+\frac{25774 \zeta(7)}{27}
\end{aligned}
$$

Numerically,

$$
b_{5}=-\left(2.57356 \times 10^{-3}\right) N^{4}+1.152827 N^{3}+72.23315 N^{2}+771.20866 N+2003.97619 .
$$

As $N$ increases from 1 , this coefficient is initially positive and is an increasing function of $N$, but it reaches a maximum at $N=374.02$ and then decreases as $N$ passes this value. As $N$ increases through the value $N=N_{b 5 z}$, where

$$
N_{b 5 z}=504.74
$$

$b_{5}$ decreases through zero to negative values, and remains negative for larger $N$ (see Table【). Here $N_{b 5 z}$ is the relevant root of the quartic equation $b_{5}=0$ (the other three roots occur occur at negative, and hence unphysical, values of $N$ ). These sign changes in $b_{4}$ and $b_{5}$ (as calculated in the $\overline{\mathrm{MS}}$ scheme) at large $N$ affect the behavior of the higher-loop $\beta$ function, as will be evident from our analysis below.

A comment is in order here concerning the generic size of higher-loop coefficients. The expansion (2.3) is a specific example of a series expansion of a generic quantity $\mathcal{O}$ in this theory, which can be written as

$$
\mathcal{O}=\sum_{n} c_{\mathcal{O}, n} a^{n}
$$

where $n$ denotes the loop order. From asymptotic estimates, it has been concluded that for $n \gg 1$, the coefficient $\left|c_{\mathcal{O}, n}\right|$ grows asymptotically dominantly as a factorial, $\sim n$ ! (with additional factors including $a^{n} n^{b}$, where $a$ and $b$ are constants) [5, 19]. Since higher-order terms are scheme-dependent, it is understood that this is the generic behavior. This forms a basis for the proof that perturbative power series expansions in this theory are not Taylor series expansions with finite radii of convergence, but instead are only asymptotic expansions. In the present theory, it is noteworthy that, as is evident from our analysis above and from Table I, there are values of $N$ for which the coefficients $b_{4}$ and $b_{5}$, as calculated in the $\overline{\mathrm{MS}}$ scheme, have zeros. For a value of $N$ where $b_{n}$ vanishes (while $b_{n-1}$ does not vanish), it will obviously not be the case that $\left|b_{n} / b_{n-1}\right|$ exhibits the generic largeorder growth as a function of $n$. Of course, since the loop orders $n=4$ and $n=5$ are not $\gg 1$, these zeros are not inconsistent with the dominant $n$ ! growth in magnitude for $n \gg 1$.

\section{F. $n$-Loop Beta Function and Associated Ratio $R_{n}$}

In Fig. 1 we plot the respective $n$-loop beta functions $\beta_{a, n \ell}$ for $n=2,3,4,5$ loops and $N=1$. This plot shows the ranges in $a$ over which the calculations of the beta function to various loop orders agree with each other. Another useful way of showing this is to plot the ratio $R_{a, n}$ of $\beta_{a, n \ell}$ divided by $\beta_{a, 1 \ell}$, as defined in Eq. (2.5), and we do this in Figs. 2 4 for the cases $N=1, N=10$, and $N=100$, respectively. Clearly,

$$
\frac{R_{n+1}}{R_{n}}=\frac{\beta_{a,(n+1) \ell}}{\beta_{a, n \ell}},
$$

so that the ratio of ratios in Eq. (2.17) measures the extent to which the $n$-loop and $(n+1)$-loop beta functions agree in value for a given $a$ and $N$.

In the case $N=1$, as is evident from Figs. 1 and 2 , $\beta_{a, 2 \ell}$ and $\beta_{a, 3 \ell}$ (equivalently, the curves for $R_{2}$ and $R_{3}$ ) are close to each other in the interval $0 \leq a \lesssim 0.04$, but as $a$ increases beyond $0.04, \beta_{a, 3 \ell}$ deviates progressively upward relative to $\beta_{a, 2 \ell}$. At higher-loop order, $\beta_{a, 3 \ell}$ and $\beta_{a, 4 \ell}$ (equivalently, the curves for $R_{3}$ and $R_{4}$ ) are close to each other in essentially the same interval $0 \leq a \lesssim 0.04$, and for larger $a, \beta_{a, 4 \ell}$ deviates below $\beta_{a, 3 \ell}$ (and eventually also below $\beta_{a, 2 \ell}$ ). Even going as high as five-loop order does not significantly increase the interval in $a$ in which the beta functions calculated to the highest two successive loop orders, namely $\beta_{a, 4 \ell}$ and $\beta_{a, 5 \ell}$ in this case, agree with each other. Numerically, the values of $\beta_{a, 4 \ell}$ and $\beta_{a, 5 \ell}$ (equivalently, the curves for $R_{4}$ and $R_{5}$ ) are close to each other only for $a$ up to about 0.05. For larger $a, \beta_{a, 5 \ell}$ deviates progressively upward relative to $\beta_{a, 4 \ell}$. An important conclusion from this analysis and from Figs. 1 14 is that the zero in the two-loop beta function (for each of the values of $N$ ) occurs at too large a value of $a$ for the perturbative calculation to be reliable. We have also established the same result for other values of $N$.

This behavior contrasts with the situation concerning an IR zero of the beta function of an asymptotically free non-Abelian gauge theory when calculated to progressively higher loop orders, up to loop order $n=4$ [20]-[24]. For example, consider an $\mathrm{SU}\left(N_{c}\right)$ gauge theory with $N_{f}$ 
fermions in the fundamental representation. As one can see from Fig. 1 in Ref. 23] for an SU(2) gauge theory with $N_{f}=8$ fermions and from Fig. 2 in [23] for an $\mathrm{SU}(3)$ gauge theory with $N_{f}=12$ fermions, the range of squared gauge couplings $\alpha=g^{2} /(4 \pi)$ over which the three-loop and four-loop beta functions for $\alpha$ agree with each other is significantly larger and extends to stronger coupling than the range of $\alpha$ for which the two-loop and three-loop beta functions are in close agreement.

\section{ZEROS OF THE BETA FUNCTION}

\section{A. $\beta_{a, 2 \ell}$}

In this section we discuss the zeros of the $n$-loop $\beta$ function, $\beta_{a, n \ell}$. Clearly, $\beta_{a, n \ell}$ has a double zero at the origin. In addition to the zero at $a=0$, as is well-known, the two-loop beta function, $\beta_{a, 2 \ell}$, has a UV zero at $a=$ $a_{U V, 2 \ell}$, where

$$
a_{U V, 2 \ell}=-\frac{b_{1}}{b_{2}}=\frac{N+8}{3 N+14} .
$$

This UV zero of $\beta_{a, 2 \ell}$ is a monotonically decreasing function of $N$ for physical $N$, which decreases from the value

$$
a_{U V, 2 \ell}=\frac{9}{17} \quad \text { at } N=1
$$

and approaches the limit

$$
\lim _{N \rightarrow \infty} a_{U V, 2 \ell}=\frac{1}{3} .
$$

As is evident from Eq. 3.1 , the corresponding value of $\lambda_{U V, 2 \ell}$ is quite large. However, since a given loop integral generically produces terms $a=\lambda /\left(16 \pi^{2}\right)$, one must examine explicit higher-loop results to judge whether this two-loop zero is a robust, reliable prediction of perturbation theory or whether, on the contrary, it occurs at too large a value of $\lambda$ to be a reliable prediction. We address this question here.

\section{B. General Methods for Analysis of Zeros of $\beta_{a, n \ell}$ for $n \geq 3$}

We proceed to calculate zeros of the $n$-loop beta function $\beta_{a, n \ell}$ for $n \geq 3$. In general, the condition that the $n$-loop beta function $\beta_{a, n \ell}$ has a zero away from the origin $a=0$ is the polynomial equation of degree $n-1$ in $a$ :

$$
\sum_{\ell=1}^{n} b_{n} a^{\ell-1}=0
$$

Although only one of the roots of Eq. (3.4) will be relevant for our analysis, it will be useful to characterize the full set of roots, as in [23]. To do this, one may make use of information from the discriminant of Eq. (3.4), denoted $\Delta_{n}\left(b_{1}, b_{2}, \ldots, b_{n+1}\right)$. Some relevant formulas on discriminants are given in Appendix A

$$
\text { C. } \beta_{a, 3 \ell}
$$

The condition that the three-loop beta function, $\beta_{a, 3 \ell}$, vanishes at a nonzero value of $a$, in addition to the IR zero at $a=0$, is the special case of Eq. (3.4) with $n=3$, viz., the quadratic equation $b_{1}+b_{2} a+b_{3} a^{2}=0$. We find that for $b_{3}$ calculated in the $\overline{\mathrm{MS}}$ scheme, this equation has no physical solutions. Formally, the solutions of this quadratic equation are

$$
a=\frac{1}{2 b_{3}}\left(-b_{2} \pm \sqrt{\Delta_{2}\left(b_{1}, b_{2}, b_{3}\right)}\right) .
$$

However, these expressions are complex, as is evident from the fact that the discriminant is negative (for all physical values of $N$ ):

$$
\begin{aligned}
\Delta_{2}\left(b_{1}, b_{2}, b_{3}\right) & =-\frac{1}{81}\left(\frac{33}{2} N^{3}+512 N^{2}+4412 N+10076\right)-\frac{16}{27}\left(5 N^{2}+62 N+176\right) \zeta(3) \\
& =-\left(0.2037 N^{3}+9.8826 N^{2}+98.6336 N+249.7651\right)
\end{aligned}
$$

Thus, with $b_{3}$ calculated in the $\overline{M S}$ scheme, $\beta_{a, 3 \ell}$ does not have any physical UV zero.
D. $\beta_{a, 4 \ell}$

The condition that $\beta_{a, 4 \ell}=0$ for $a \neq 0$ is the $n=4$ special case of Eq. (3.4), namely, the cubic equation

$$
b_{1}+b_{2} a+b_{3} a^{2}+b_{4} a^{3}=0
$$


The nature of the roots of Eq. (3.7) is determined by the sign of the discriminant,

$$
\begin{aligned}
\Delta_{3} \equiv \Delta_{3}\left(b_{1}, b_{2}, b_{3}, b_{4}\right) & =b_{2}^{2} b_{3}^{2}-27 b_{1}^{2} b_{4}^{2}-4\left(b_{1} b_{3}^{3}+b_{4} b_{2}^{3}\right) \\
& +18 b_{1} b_{2} b_{3} b_{4}
\end{aligned}
$$

The following properties of $\Delta_{3}$ will be useful here [25]: (i) if $\Delta_{3}>0$, then all of the roots of Eq. (3.7) are real; (ii) if $\Delta_{3}<0$, then Eq. (3.7) has one real root and a complex-conjugate pair of roots; (iii) if $\Delta_{3}=0$, then at least two of the roots of Eq. (3.7) coincide. We find that, with $b_{3}$ and $b_{4}$ calculated in the $\overline{\mathrm{MS}}$ scheme, $\Delta_{3}\left(b_{1}, b_{2}, b_{3}, b_{4}\right)$ (which is a polynomial of degree 8 in $N$ ) is negative for all physical $N$. Hence, the solutions to Eq. (3.7) consist of one real root and a complex-conjugate pair of roots. We display values of the real root $a_{U V, 4 \ell}$ for various values of $N$ in Table II. We find that as $N$ increases from 1 to $N \simeq 770$, the real root decreases from 0.233 to approximately 0.0714 , but then increases again and diverges as $N \nearrow N_{b 4 z}=3218.755$, where $b_{4}$ vanishes. For $N \geq N_{b 4 z}, \beta_{a, 4 \ell}$ has no physical UV zero. (The positive real root that diverged as $N \nearrow N_{b 4 z}$ now occurs at negative real values for this range of $N$, and the other two roots of Eq. (3.7) continue to be a complexconjugate pair.)

\section{E. $\beta_{a, 5 \ell}$}

The condition for a zero of $\beta_{a, 5 \ell}$ with $a \neq 0$ is the special case of Eq. (3.4) with $n=5$, namely, the quartic equation

$$
b_{1}+b_{2} a+b_{3} a^{2}+b_{4} a^{3}+b_{5} a^{4}=0 \text {. }
$$

The discriminant, $\Delta_{4} \equiv \Delta_{4}\left(b_{1}, b_{2}, b_{3}, b_{4}, b_{5}\right)$, of this equation is given by Eqs. (A9) and (A3) in Appendix A. We have calculated this and found that it is positive for all physical $N$ except for the interval

$$
493.096<N<504.740
$$

We denote the lower end of this interval as $N_{\Delta_{4} z}=$ 493.096. For the interval of $N$ from 1 to $N_{\Delta_{4} z}$, Eq. (3.9) has no physical solutions. As $N$ (analytically continued from the positive integers to the reals) increases through the value $N_{\Delta_{4} z}$, a double real root of Eq. (3.9) appears at $a=0.1264$ and then bifurcates into two real roots. The smaller of these is the physical $a_{U V, 5 \ell}$ and decreases below 0.1264 as $N$ increases beyond $N_{\Delta_{4} z}$, while the larger root increases above $a=0.1264$ as $N$ increases above $N_{\Delta_{4} z}$. As $N$ (again, considered as a real variable) approaches the value $N=504.740$ from below, the larger real root diverges, leaving only the lower one. This continues to decrease as $N$ increases further. We list values of $a_{U V, 5 \ell}$ for various $N$ in Table II.

\section{F. Comparison of Calculations to Different Loop Orders}

A necessary condition for a perturbative calculation of the beta function $\beta_{a}$ to be reliable is that the fractional change

$$
\left|\frac{\beta_{a, n+1}-\beta_{a, n}}{\beta_{a, n}}\right|
$$

should generally decrease as the loop order $n$ increases, at least away from a zero of $\beta_{a, n}$. Another necessary condition for the reliability of a result on a zero of the $n$ loop beta function, $\beta_{a, n}$, is that when one calculates the beta function to the next higher-loop order, viz., $\beta_{a, n+1}$, the zero should still be present and its value should not shift very much. For the specific case at hand, where we are investigating a possible UV zero of $\beta_{a}$, this condition is that the fractional shift

$$
\frac{\left|a_{U V, n+1}-a_{U V, n}\right|}{a_{U V, n}}
$$

should be small. Our calculations above show that neither of these two necessary conditions is satisfied for this theory. As was evident from our plots of the $n$-loop beta functions and the ratios $R_{n}$ of the $n$-loop beta function divided by the one-loop beta function given above, the fractional change (3.11) is not small for the values of $a$ that are relevant for the analysis of a possible UV zero, even for the highest loop order $n$ that we have investigated. Recall from Eqs. (3.1) - (3.3) that the values of $a_{U V, 2 \ell}$ range from $9 / 17$ to $1 / 3$ as $N$ increases from 1 to $\infty$. Furthermore, although the two-loop beta function $\beta_{a, 2 \ell}$ exhibits a UV zero, this is absent in the three-loop beta function $\beta_{a, 3 \ell}$ and although the four-loop and five loop beta functions have UV zeros for certain ranges of $N$, they occur at rather different values than for $\beta_{a, 2 \ell}$. For example, for $N=1, a_{U V, 4 \ell}=0.233$, which is substantially smaller than $a_{U V, 2 \ell}=0.529$, and neither the three-loop nor five-loop beta function has a UV zero. A similar situation holds for $N=100$. For $N=1000$, $a_{U V, 4 \ell}=0.0724$, which again is considerably smaller than $a_{U V, 2 \ell}=0.334$, and $\beta_{a, 3 \ell}$ has no UV zero, while $a_{U V, 5 \ell}=0.0228$, which is a substantially different value than both the two-loop and four-loop UV zeros. Similar comments apply for other values of $N$. Our higher-loop analysis therefore leads us to conclude that the (perturbatively calculated) beta function of this theory does not exhibit a robust, reliably calculable UV zero to the highest loop order, namely five loops, to which it has been computed.

\section{LARGE- $N$ LIMIT}

Further insight into the question of a UV zero of the beta function of this theory can be obtained from an 
analysis of the limit

$$
N \rightarrow \infty, \text { with } x(\mu) \equiv N a(\mu) \text { a finite function of } \mu .
$$

We denote this as the LN limit and will use the symbol $\lim _{L N}$ to refer to it. For the purpose of this analysis, we define a rescaled beta function that is finite in the LN limit. For large $N$, the two scheme-independent coefficients have the asymptotic behavior $b_{1} \sim N / 3$ and $b_{2} \sim-N$, while $b_{\ell} \sim$ const. $\times N^{\ell-1}$ for $\ell \geq 3$ for the higher-loop coefficients that have been calculated in the $\overline{\mathrm{MS}}$ scheme. From Eq. (2.6) one can write

$$
b_{1}=b_{1,1} N+b_{1,0} \quad \text { where } b_{1,1}=\frac{1}{3}, \quad b_{1,0}=\frac{8}{3} .
$$

We thus extract the leading- $N$ factors and define

$$
\check{b}_{\ell}=\lim _{L N} \frac{b_{\ell}}{N^{\ell-1}} \quad \text { for } \ell \geq 2 .
$$

so that these $\check{b}_{\ell}$ are finite in the large- $N$ limit. The explicit values of the $\breve{b}_{\ell}$ follow from the expressions given above for the $b_{\ell}$; thus, $\breve{b}_{2}=-1, \breve{b}_{3}=11 / 72$, etc. Since the LN limit is defined so that $x(\mu)$ is a finite function of $\mu$, the appropriate beta function that is finite in this limit is

$$
\begin{aligned}
\beta_{x} & =\frac{d x}{d t}=\lim _{L N} N \beta_{a} \\
& =x^{2}\left[b_{1,1}+\frac{1}{N} \sum_{\ell=2}^{\infty} \check{b}_{\ell} x^{\ell-1}\right] .
\end{aligned}
$$

The $n$-loop beta function in the LN limit, denoted $\beta_{x, n \ell}$, is defined via Eq. (4.4) with the upper limit on the sum being $\ell=n$ rather than $\ell=\infty$. From Eq. (4.4), it is evident that in the LN limit, for any given loop order $n, \beta_{x, n \ell}$ has no UV zero $x_{U V, n \ell}$, since

$$
\lim _{L N} \frac{1}{N} \sum_{\ell=2}^{n}\left(\frac{\check{b}_{\ell}}{b_{1,1}}\right) x^{\ell-1}=0 .
$$

Hence, in the $N \rightarrow \infty$ limit, as $\mu$ increases, $x(\mu)$ increases, eventually exceeding the range of values where the perturbative $n$-loop expansion of $\beta_{x, n \ell}$ is reliable. This result in the LN limit agrees with our specific calculations for large finite values of $N$ as shown in Table II]

\section{EFFECT OF SCHEME TRANSFORMATIONS}

In view of the fact that the $b_{\ell}$ with $\ell \geq 3$ are schemedependent, one is motivated to study the effect of a scheme transformation on the beta function of this $\lambda|\vec{\phi}|_{4}^{4}$ theory. It should be recalled that scheme dependence is also present, e.g., in higher-loop perturbative calculations in quantum chromodynamics (QCD) and does not prevent one from using such calculations successfully in comparisons with data [26]. If one were interested in a zero of the beta function at zero coupling, as with the UV fixed point of an asymptotically free gauge theory like QCD [27] or the IR fixed point of the $\lambda|\vec{\phi}|_{4}^{4}$ theory, then one would expect that it should be possible to transform away the terms in the beta function at loop order $\ell \geq 3$, and an explicit construction that does this was presented in [28]. However, it was also pointed out in [28] that it is considerably more difficult to construct an acceptable scheme transformation that removes some set of coefficients at loop order 3 or higher at a zero of the beta function away from the origin in coupling constant space than it is at the origin. Ref. [28] gave a set of conditions that such a scheme transformation must satisfy to be physically acceptable. Here we recall the basic formalism; we refer the reader to [28]-[30] for further details (see also [31]).

A scheme transformation can be expressed as a mapping between $\lambda$ and $\lambda^{\prime}$, or equivalently, $a$ and $a^{\prime}$, which we write as $a=a^{\prime} f\left(a^{\prime}\right)$. We will refer to $f\left(a^{\prime}\right)$ as the scheme transformation function. Since scheme transformations cannot change the theory in the limit where the coupling goes to zero, one requires that $f(0)=1$. We expand $f\left(a^{\prime}\right)$ as a power series of the form

$$
f\left(a^{\prime}\right)=1+\sum_{s=1}^{s_{\max }} k_{s}\left(a^{\prime}\right)^{s},
$$

where the $k_{s}$ are constants, and, a priori, $s_{\max }$ may be finite or infinite. After the scheme transformation is applied, the beta function in the new scheme has the form (2.3) with $a$ replaced by $a^{\prime}$ and $b_{\ell}$ replaced by $b_{\ell}^{\prime}$. Expressions for the $b_{\ell}^{\prime}$ in terms of the $b_{\ell}$ and $k_{s}$ were given in [28]. Ref. [30] presented a generalized one-parameter family of scheme transformation denoted $S_{R, m, k_{1}}$ with $m \geq 2$ and $s_{\max }=m$ that can render $b_{\ell}^{\prime}=0$ for $3 \leq \ell \leq m+1$ inclusive and can be applied at a zero of the beta function away from the origin.

A natural approach is to investigate the effect of applying scheme transformations in the one-parameter family $S_{R, 2, k_{1}}$ to the beta function in the $\overline{\mathrm{MS}}$ scheme in order to render $b_{3}^{\prime}=0$ in the transformed scheme. This family of scheme transformations depends on a parameter $k_{1}$ and has

$$
k_{2}=\frac{b_{3}}{b_{1}}+\frac{b_{2}}{b_{1}} k_{1}+k_{1}^{2}
$$

(with $k_{s}=0$ for $s \geq 3$ ). If this scheme transformation were to be applicable, then in the transformed scheme the beta function would have the form, for $n=3$,

$$
\beta_{a^{\prime}, 3 \ell}=\left(a^{\prime}\right)^{2}\left[b_{1}+b_{2} a^{\prime}\right]
$$

and, for $n \geq 4$,

$$
\beta_{a^{\prime}, n \ell}=\left(a^{\prime}\right)^{2}\left[b_{1}+b_{2} a^{\prime}+\sum_{\ell=4}^{n} b_{\ell}^{\prime}\left(a^{\prime}\right)^{\ell-1}\right] \quad \text { for } n \geq 4 .
$$


One of the necessary conditions for the acceptability of the scheme transformation is that $f\left(a^{\prime}\right)$ must be positive and not too different from unity, since otherwise the transformation or its inverse would map a reasonably small value of the coupling, for which perturbative methods could be reliable, to an excessively large value, beyond the region where these methods could be reliably applied. In particular, this condition must be satisfied at (and in the neighborhood of) the scheme-independent value $a=a_{U V, 2 \ell}=a_{U V, 2 \ell}^{\prime}$. We thus consider the evaluation of $f\left(a^{\prime}\right)$ at this point. We have

$$
f\left(a_{U V, 2 \ell}^{\prime}\right)=1+\frac{b_{1} b_{3}}{b_{2}^{2}}+\frac{b_{1}^{2}}{b_{2}^{2}} k_{1}^{2},
$$

where $b_{3}$ is calculated in the $\overline{\mathrm{MS}}$ scheme. In addition to the first term, both the second and third terms on the right-hand side of Eq. (5.5) are positive in the $\overline{\mathrm{MS}}$ scheme, since $b_{3}$ is positive. Given that the coefficient of $k_{1}^{2}$ is positive, it follows that $f\left(a_{U V, 2 \ell}^{\prime}\right)$ is minimized by taking $k_{1}=0$. We thus choose $k_{1}=0$, so that $S_{R, 2, k_{1}}$ reduces to the $S_{R, 2}$ transformation [28]-30]. Even with this choice, we find that as $N$ increases, $f\left(a_{U V, 2 \ell}^{\prime}\right)$ quickly becomes excessively large, preventing one from using this scheme transformation over a very large interval of values of $N$. A second condition is that the Jacobian $d a / d a^{\prime}$ should not approach or equal zero, since otherwise the scheme transformation is singular. As with $f\left(a^{\prime}\right)$, we require this condition to be satisfied at (and in the neighborhood of) $a=a_{U V, 2 \ell}=a_{U V, 2 \ell}^{\prime}$. At this point,

$$
J=1+\frac{3 b_{1} b_{3}}{b_{2}^{2}}+\frac{b_{1}}{b_{2}} k_{1}+\frac{3 b_{1}^{2}}{b_{2}^{2}} k_{1}^{2} .
$$

Since the term $3 b_{1} b_{3} / b_{2}^{2}$ is positive, the choice that we have made, namely $k_{1}=0$, also guarantees that $J$ stays positive.

By construction, after application of the $S_{R, 2, k_{1}}$ scheme transformation, the three-loop beta function $\beta_{a^{\prime}, 3 \ell}$, has a UV zero, in contrast to the situation in the original $\overline{\mathrm{MS}}$ scheme, where it does not. This is evident from the $n=3$ special case of Eq. (5.4). Since $b_{3}^{\prime}=0$, this UV zero occurs at the same value of $a^{\prime}$ as the two-loop value, i.e.,

$$
a_{U V, 3 \ell}^{\prime}=a_{U V, 2 \ell}^{\prime}=a_{U V, 2 \ell}=-\frac{b_{1}}{b_{2}} .
$$

However, in order for one to take this as a significant indication that there is, in fact, a UV zero in the beta function, it must continue to be present when calculated to higher order (at least for the range of $N$ where this $S_{R, 2}$ scheme transformation can be applied) and at higher orders, the fractional change in the value should decrease to be reasonably small values.

To investigate whether these conditions are satisfied, we have calculated the four-loop and five-loop beta functions in the $S_{R, 2}$-transformed scheme, $\beta_{a^{\prime}, 4 \ell}$ and $\beta_{a^{\prime}, 5 \ell}$ and have investigated their zeros. For this calculation we use the expressions for $b_{4}^{\prime}$ and $b_{5}^{\prime}$ from [28]-[30]. A UV zero of the four-loop beta function in the transformed scheme, $\beta_{a^{\prime}, 4 \ell}$, is given by the relevant root of the cubic equation $b_{1}+b_{2} a^{\prime}+b_{4}^{\prime}\left(a^{\prime}\right)^{3}=0$, namely the positive root nearest to the origin. Similarly, a UV zero of $\beta_{a^{\prime}, 4 \ell}$, would be given by the relevant root of the quartic equation $b_{1}+b_{2} a^{\prime}+b_{4}^{\prime}\left(a^{\prime}\right)^{3}+b_{5}^{\prime}\left(a^{\prime}\right)^{4}=0$, namely a positive root nearest to the origin. We show the results in Table III for the range of $N$ where $f\left(a_{U V, 2 \ell}^{\prime}\right)$ is not excessively large, namely $1 \leq N \lesssim 10$. For illustration, we also show calculations for the value $N=100$, although $f\left(a^{\prime}\right)$ is arguably too large for the scheme transformation to be applicable at this value of $N$. As is evident from Table III, although $\beta_{a^{\prime}, 4 \ell}$ has a UV zero, it occurs at a considerably smaller value of $a^{\prime}$ than the two-loop value, $a_{U V, 2 \ell}^{\prime}$. Furthermore, we find that at five-loop order the beta function in the transformed scheme, $\beta_{a^{\prime}, 5 \ell}$, does not have a (physical) UV zero. (It has two pairs of complexconjugate roots). Therefore, over the interval of $N$ where the $S_{R, 2}$ scheme transformation can be applied without excessively large $f\left(a^{\prime}\right)$, the necessary conditions stated above for these results to be consistent with a robust UV zero of the beta function are not satisfied; i.e., although $\beta_{a^{\prime}, 4 \ell}$ has a UV zero, it occurs at a considerably smaller value of $a^{\prime}$ than $a_{U V, 2 \ell}^{\prime}$ and, furthermore, $\beta_{a^{\prime}, 5 \ell}$ does not have a UV zero. By continuity, our results also apply for an (infinite) set of other $S_{R, 2, k_{1}}$ scheme transformations whose functions $f\left(a^{\prime}\right)$ are close to the function $f\left(a^{\prime}\right)$ for $S_{R, 2}$, namely the set with small $\left|k_{1}\right|$.

Our results from the analysis of the beta function up to five-loop order after application of the $S_{R, 2, k_{1}}$ scheme transformations thus agree with our results from the analysis in the original $\overline{\mathrm{MS}}$ scheme; in both studies, we do not find evidence that the beta function of this theory has a UV zero, at least insofar as we can use perturbative methods reliably to investigate it. Indeed, this was already clear from Figs. 1,4. These showed that although the two-loop beta function has a UV zero, this occurs at a value $a_{U V, 2 \ell}$ (dependent on $N$ ) that is well beyond the range where the perturbative calculation is reliable, since the respective higher-loop beta functions $\beta_{a, n \ell}$ with $n=3,4,5$ loops differ considerably from $\beta_{a, 2 \ell}$ for $a \simeq a_{U V, 2 \ell}$. The absence of a reliably calculable UV zero of $\beta_{a, n \ell}$ means that, to the highest loop order, namely $n=5$ loops, to which the beta function has been calculated, $\lambda(\mu)$ increases with increasing $\mu$, eventually exceeding the range where perturbative methods of analysis can be used. Some recent discussions of possibilities for the nonperturbative behavior of $\lambda|\vec{\phi}|_{4}^{4}$ theory include [5, 32].

A scheme transformation constitutes one type of resummation of a perturbation series. A different method of analysis is provided by Padé approximants [33]. We calculate and analyze these next. 


\section{ANALYSIS WITH PADÉ APPROXIMANTS}

Given a series for an abstract function $f(z)=$ $\sum_{s=0}^{m} c_{s} z^{s}$, the $[p, q]$ Padé approximant is the rational function

$$
[p, q]=\frac{\sum_{j=0}^{p} N_{j} z^{j}}{\sum_{k=0}^{q} D_{k} z^{k}}
$$

with polynomials in the numerator and denominator of degree $p$ and $q$, respectively, where $p+q=m$ [33]. Without loss of generality, one may take $D_{0}=1$, so that $N_{0}=c_{0}$. The coefficients $N_{j}$ with $j=1, \ldots, p$ and $D_{k}$ with $k=1, \ldots, q$ are determined by the $m$ coefficients $c_{1}, \ldots, c_{m}$. Thus, a $[p, q]$ Padé approximant to $f(z)$ is a closed-form rational approximation whose Taylor series expansion in $z$ matches the power series for $f(z)$ to the highest order to which it is calculated.

It is natural to inquire how the zeros of various Padé approximants to $\beta_{a, n \ell}$ compare with those of $\beta_{a, n \ell}$, as we did, e.g., in [21] for the IR zero of the beta function of a non-Abelian gauge theory. For this purpose, it is convenient to extract the overall factor of $a^{2}$ in $\beta_{a, n \ell}$ and thus compute the Padé approximants to the function $\beta_{a, n \ell} / a^{2}=b_{1}+\sum_{\ell=2}^{n} b_{\ell} a^{\ell-1}$. At a given loop order $n$, we can calculate the $[p, q]$ Padé approximants with $p+q=n-1$. The $[n-1,0]$ Padé approximant to $\beta_{a, n \ell} / a^{2}$ is the function itself, and the $[0, n-1]$ approximant has no zeros so we do not consider these. The use of Padé approximants to scattering amplitudes has a long history in particle physics and, as usual, the fact that the Padé approximant has a Taylor series expansion with a finite radius of convergence is not to be taken as implying that the actual function being approximated (in this case, $\beta_{n \ell} / a^{2}$ ) has such a Taylor series expansion in powers of the coupling; indeed, it is known that the series expansion (2.3) is only an asymptotic expansion [19, 34]. Clearly, there are several necessary conditions for a zero of a $[p, q]$ Padé approximant to $\beta_{n \ell} / a^{2}$ to be taken to be physically meaningful. Two of these conditions are (i) that this zero must occur on the positive real axis in the complex $a$ plane at a value that is not too different from $a_{U V, 2 \ell}$; (ii) the location of the zero must be closer to the origin $a=0$ than any of the $q$ poles. Moreover, no implication is made that these approximants accurately describe the large- $a$ behavior of the beta function (which would be $\beta_{n, \ell} \propto a^{2+p-q}$ ).

We have carried out this analysis with Padé approximants and have found that it confirms the conclusions that we reached from our study of the $n$-loop beta function $\beta_{a, n \ell}$ itself. For example, let us consider the case $N=1$. As with other values of $N$, the three-loop beta function, $\beta_{a, 3 \ell}$, has no UV zero. (Aside from the double zero at $a=0$, it has a complex-conjugate pair of zeros at $a=0.08705 \pm 0.29084 i$.) Similarly, the relevant Padé approximant to $\beta_{a, 3 \ell} / a^{2}$, namely $[1,1]$, has only an unphysical zero at $a=-0.2594$ (as well as a pole at an unphysical point even closer to the origin, namely $a=-0.19745$ ). At the four-loop level, $\beta_{a, 4 \ell}$ has a UV zero at $a=0.2333$, but this is not reproduced by either of the relevant Padé approximants to $\beta_{a, 4 \ell} / a^{2}$; the $[1,2]$ approximant has an unphysical zero at $a=-0.1294$ (and unphysical poles at $a=-0.1138$ and $a=-1.2005)$, while the $[2,1]$ approximant has an unphysical zero at $a=-0.1400$ and a UV zero at $a=1.4543$, much larger than the UV zero of $\beta_{a, 4 \ell}$ (as well as a pole nearer to the origin, at the unphysical value $a=-0.1198)$.

At the five-loop level, $\beta_{a, 5 \ell}$ has no physical UV zero. (In addition to the double zero at $a=0$, it has zeros at the two complex-conjugate pairs $a=-0.09440 \pm 0.14585 i$ and $a=0.1421 \pm 0.1213 i)$. Of the three relevant Padé approximants to $\beta_{a, 5 \ell} / a^{2}$, the [1,3] approximant has a zero at $a=-0.0949$ (and poles at $a=-0.0899, a=-0.4644$, and $a=1.1714) ;[2,2]$ has zeros at $a=-0.0874$ and $a=-0.5298$ (and poles at $a=-0.0840$ and $a=$ $-0.3013)$, and $[3,1]$ has zeros at $a=-0.10245$ and $a=0.2439 \pm 0.6002 i$ (and a pole at $a=-0.09535$ ). Evidently, all of the zeros of these Padee approximants are unphysical.

We find similar results for other values of $N$. Thus, from our calculation and analysis of Padé approximants to the $n$-loop beta function, we add to our evidence against a stable, reliably calculable UV zero in the $\lambda|\vec{\phi}|_{4}^{4}$ theory. Other resummation methods such as a Borel transform could also be applied, but the findings from these two methods, namely scheme transformations and Padé approximants already provide strong evidence against a robust UV zero in the beta function for this theory up to the five-loop order.

\section{DISCUSSION}

In this section we compare our present results with those on zeros of the beta function for some other theories. We begin with the nonlinear $\sigma$ model in $d=2+\epsilon$ dimensions [10]. Both the $\lambda|\vec{\phi}|_{4}^{4}$ theory and the nonlinear $\sigma$ model in $d=2+\epsilon$ are IR-free, but in the NL $\sigma \mathrm{M}$ one can choose a parameter, namely $\epsilon$, to approach zero so as to make the UV fixed point occur at an arbitrarily weak coupling. In this $\mathrm{NL} \sigma \mathrm{M}$ let us define appropriately rescaled quantities $\tilde{\lambda} \equiv \lambda N$ and $\beta_{\tilde{\lambda}}=d \tilde{\lambda} / d t$, so that Eq. (1.4) with Eq. (1.5) reads

$$
\beta_{\tilde{\lambda}}=\epsilon \tilde{\lambda}\left(1-\frac{\tilde{\lambda}}{\tilde{\lambda}_{c}}\right)
$$

where $\tilde{\lambda}_{c}=2 \pi \epsilon$. As noted before, this result was obtained by summing an infinite set of Feynman diagrams that dominate in the large- $N$ limit. By letting $\epsilon \rightarrow 0^{+}$, one can make the UVFP occur at an arbitrarily small value of $\tilde{\lambda}_{c}$.

A different but related comparison can be made with the calculation of the IR zero of the beta function for the gauge coupling, $\beta_{\alpha}=d \alpha / d t$ (where $\alpha=g^{2} /(4 \pi)$ ), that is present in an asymptotically free vectorial non-Abelian gauge theory in $d=4$ dimensions with gauge group $G$ 
and an appropriately large fermion content. Thus, consider such a theory with $N_{f}$ copies of massless fermions transforming according to a representation $R$ of $G$ and denote $N_{f, b 1 z}$ as the upper bound on $N_{f}$ for asymptotic freedom and $N_{f, b 2 z}$ as the value of $N_{f}$ such that for $N_{f}>N_{f, b 2 z}$, the one-loop and two-loop terms in $\beta_{\alpha}$ have opposite sign. Since $N_{f, b 2 z}<N_{f, b 1 z}$, it follows that there is an interval in $N_{f}$, namely $N_{f, b 2 z}<N_{f}<N_{f, b 1 z}$ for which the two-loop $\beta_{\alpha}$ function has an IR zero. Importantly, all of the $n$-loop beta functions for $n=2,3,4$ loop orders consistently exhibit an IR zero, and the fractional shift in this IR zero is reduced when one compares the beta functions at $n=3$ and $n=4$ loop order, as contrasted with the shift between the beta functions at $n=2$ and $n=3$ loop order. This is evident from Figs. 1 and 2 of Ref. 23]. Furthermore, with appropriate choices of $G$, $R$, and $N_{f}$, one can make this IR zero occur at a small value of $\alpha$ [35]. In particular, consider an asymptotically free vectorial gauge theory with $G=\mathrm{SU}\left(N_{c}\right), R$ equal to the fundamental representation, $N_{c} \rightarrow \infty, N_{f} \rightarrow \infty$, with $r=N_{f} / N_{c}$ fixed, and $\xi(\mu) \equiv \alpha(\mu)^{2} N_{c}$ a finite function of $\mu$. Then for $r$ in the interval $34 / 13<r<11 / 2$, the rescaled beta function $\beta_{\xi}=d \xi / d t$ has an IR zero at the two-loop level at

$$
\xi_{U V, 2 \ell}=\frac{4 \pi(11-2 r)}{13 r-34} .
$$

The value of $\xi_{U V, 2 \ell}$ can be made arbitrarily small by letting $r$ approach 11/2 from below. The situation in the $\lambda|\vec{\phi}|_{4}^{4}$ theory is fundamentally different from that in this non-Abelian gauge theory because there is no parameter that can be tuned to make the two-loop value of $a_{U V, 2 \ell} \ll 1$ or, in the large- $N$ limit, the value of $x_{U V, 2 \ell} \ll 1$. This is similar to the situation in the $\mathrm{U}(1)$ gauge theory in $d=4$ with $N_{f}$ copies of a charged fermion, where we found evidence against a UVFP in [8] (see also [9]).

In the present work we have focused on a simple $\mathrm{O}(N)$ scalar field theory involving a single coupling, using calculations to the rather high order of five loops. This is complementary to studies of more complicated theories with more than one coupling, involving RG calculations to lower than five-loop order. In passing, we add a few comments on these theories. The RG behaviors of theories with scalar and fermion fields have been studied for many years, both perturbatively and nonperturbatively (for references to the literature, see, e.g., [36]). For small values of the quartic scalar coupling $\lambda$ and the Yukawa coupling $y$, both $\beta_{\lambda}=d \lambda / d t$ and $\beta_{y}=d y / d t$ are positive, so that near the origin $(\lambda, y)=(0,0)$, as $\mu$ decreases, the RG flow is toward to origin, i.e. a free theory. One may investigate such theories for possible fixed points of the renormalization group away from the origin. For sufficiently large couplings, one must use nonperturbative methods, such as lattice simulations [37]. Studies have also been performed of models with gauge, fermion, and scalar fields, and hence three or more couplings [38, 39]. In some cases, UV-stable fixed points have been reported (e.g., 39] and references therein), motivating continued interest in the phenomenon of asymptotic safety. For the actual Standard Model, an intriguing feature is that because of the the large top-quark Yukawa coupling, instead of increasing for large $\mu$ in the ultraviolet, the quartic Higgs coupling actually decreases and eventually vanishes [40], although this is sensitive to ultraviolet completions of the Standard Model.

\section{CONCLUSIONS}

In this paper we have investigated whether there is a reliably calculable ultraviolet zero of the beta function in the $\mathrm{O}(N) \lambda|\vec{\phi}|_{4}^{4}$ field theory. This question is of interest since the two-loop beta function does have a UV zero. We have examined whether two necessary conditions are met, namely that the existence of this UV zero persists to higher-loop order and that higherorder $n$-loop calculations yield reasonably stable values of $a_{U V, n \ell}=\lambda_{U V, n \ell} /\left(16 \pi^{2}\right)$. We have carried out this study using calculations of the $n$-loop beta functions $\beta_{a, n \ell}$ for three-, four-, and five-loop order in the $\overline{\mathrm{MS}}$ scheme. We have shown that in this scheme, (i) $\beta_{\lambda, 3 \ell}$ has no UV zero; (ii) $\beta_{\lambda, 4 \ell}$ has a UV zero only for a limited range of $N$, and has no UV zero for sufficiently large $N$; (iii) $\beta_{\lambda, 5 \ell}$ has no UV zero for $N$ from 1 to almost 500, and although it does have a UV zero for larger values of $N$, this zero occurs at quite a different value of $a$ than two-loop UV zero. Thus, we find that neither of the two necessary conditions for a robust, reliably calculable UV zero of the beta function is satisfied for any $N$. This inference is confirmed by our study of the effect of applying scheme transformations to the beta function and from calculations of Padé approximants. Evidently, the zero in $\beta_{a, 2 \ell}$ occurs at too large a value for the two-loop perturbative calculation to be accurate. We thus conclude that in the range of quartic coupling $\lambda$, or equivalently, $a$, where the perturbative calculation of $\beta_{\lambda, n \ell}$ is reliable, the $\mathrm{O}(N) \lambda|\vec{\phi}|_{4}^{4}$ theory does not exhibit robust evidence of a UV zero up to the level of $n=5$ loops for any $N$. This conclusion is in accord with the current view of the $\mathrm{O}(N) \lambda|\vec{\phi}|^{4}$ theory in $d=4$ dimensions as an effective field theory, to be applied only over a restricted range of momentum scales $\mu$.

\section{Acknowledgments}

This research was partly supported by NSF grant PHY-13-16617.

\section{Appendix A: Discriminants}

Our study of the zeros of the $n$-loop beta function of the $\lambda|\vec{\phi}|_{4}^{4}$ theory requires an analysis of the zeros of the polynomial equation (3.4), of degree $n-1$ in the variable $a$ 
given by Eq. (2.2). At the loop order $\ell \geq 3$, this analysis is considerably expedited by the use of the corresponding discriminant.

Consider the polynomial of degree $m$ in an abstract variable $z$,

$$
P_{m}(z)=\sum_{s=0}^{m} c_{s} z^{s}
$$

and denote the set of $m$ roots of the equation $P_{m}(z)=$ 0 as $\left\{z_{1}, \ldots, z_{m}\right\}$. The discriminant of this equation is defined as [25]

$$
\Delta_{m} \equiv\left[c_{m}^{m-1} \prod_{i<j}\left(z_{i}-z_{j}\right)\right]^{2}
$$

Since $\Delta_{m}$ is a symmetric polynomial in the roots of the equation $P_{m}(z)=0$ (being proportional to the square of the Vandermonde polynomial of these roots), the symmetric function theorem implies that it can be expressed as a polynomial in the coefficients of $P_{m}(z)$ [41]. We will sometimes indicate this dependence explicitly, writing $\Delta_{m}\left(c_{0}, \ldots, c_{m}\right)$. The discriminant $\Delta_{m}$ is a homogeneous polynomial of degree $m(m-1)$ in the roots $\left\{z_{i}\right\}$. For the application to the analysis of the roots of Eq. (3.4), the correspondence is $z=a$ and

$$
c_{s}=b_{s+1}, \quad s=0, \ldots, m .
$$

To analyze the zeros of $\beta_{a, n \ell}$ away from the origin, given by the roots of Eq. (3.4), of degree $m=n-1$, we will thus use the discriminant $\Delta_{n-1}\left(b_{1}, b_{2}, \ldots, b_{n}\right)$. Because of the homogeneity properties of this discriminant,

$\Delta_{n-1}\left(\bar{b}_{1}, \bar{b}_{2}, \ldots, \bar{b}_{n}\right)=(4 \pi)^{-(n+1)(n-2)} \Delta_{n-1}\left(b_{1}, b_{2}, \ldots, b_{n}\right)$.
The discriminant $\Delta_{m}$ is most conveniently calculated in terms of the Sylvester matrix of $P(z)$ and $d P(z) / d z$, equivalent to the resultant matrix, denoted $S_{P, P^{\prime}}$, of dimension $(2 m-1) \times(2 m-1)[25]$ :

$$
\Delta_{m}=(-1)^{m(m-1) / 2} c_{m}^{-1} \operatorname{det}\left(S_{P, P^{\prime}}\right) .
$$

For our analysis, we use $\Delta_{m}$ for $m=2,, 3,4$. The $m=2$ discriminant, $\Delta_{2}$, is elementary: $\Delta_{2}=c_{1}^{2}-4 c_{0} c_{2}$. For $m=3$,

$$
S_{P_{3}, P_{3}^{\prime}}=\left(\begin{array}{ccccc}
c_{3} & c_{2} & c_{1} & c_{0} & 0 \\
0 & c_{3} & c_{2} & c_{1} & c_{0} \\
3 & 2 c_{2} & c_{1} & 0 & 0 \\
0 & 3 c_{3} & 2 c_{2} & c_{1} & 0 \\
0 & 0 & 3 c_{2} & 2 c_{2} & c_{1}
\end{array}\right)
$$

so that

$$
\begin{aligned}
\Delta_{3}\left(c_{0}, c_{1}, c_{2}, c_{3}\right) & =c_{1}^{2} c_{2}^{2}-27 c_{0}^{2} c_{3}^{2}-4\left(c_{0} c_{2}^{3}+c_{3} c_{1}^{3}\right) \\
& +18 c_{0} c_{1} c_{2} c_{3} .
\end{aligned}
$$$$
\text { For } m=4 \text {, }
$$

$$
S_{P_{4}, P_{4}^{\prime}}=\left(\begin{array}{ccccccc}
c_{4} & c_{3} & c_{2} & c_{1} & c_{0} & 0 & 0 \\
0 & c_{4} & c_{3} & c_{2} & c_{1} & c_{0} & 0 \\
0 & 0 & c_{4} & c_{3} & c_{2} & c_{1} & c_{0} \\
4 c_{4} & 3 c_{3} & 2 c_{2} & c_{1} & 0 & 0 & 0 \\
0 & 4 c_{4} & 3 c_{3} & 2 c_{2} & c_{1} & 0 & 0 \\
0 & 0 & 4 c_{4} & 3 c_{3} & 2 c_{2} & c_{1} & 0 \\
0 & 0 & 0 & 4 c_{4} & 3 c_{3} & 2 c_{2} & c_{1}
\end{array}\right)
$$

so that

$$
\begin{aligned}
\Delta_{4}\left(c_{0}, c_{1}, c_{2}, c_{3}, c_{4}\right) & =c_{1}^{2} c_{2}^{2} c_{3}^{2}-128 c_{0}^{2} c_{2}^{2} c_{4}^{2}-4 c_{1}^{3} c_{3}^{3}+256 c_{0}^{3} c_{4}^{3}-27\left(c_{0}^{2} c_{3}^{4}+c_{1}^{4} c_{4}^{2}\right)-4\left(c_{0} c_{2}^{3} c_{3}^{2}+c_{1}^{2} c_{2}^{3} c_{4}\right) \\
& +18\left(c_{0} c_{1} c_{2} c_{3}^{3}+c_{1}^{3} c_{2} c_{3} c_{4}\right)-6 c_{0} c_{1}^{2} c_{3}^{2} c_{4}+144\left(c_{0} c_{1}^{2} c_{2} c_{4}^{2}+c_{0}^{2} c_{2} c_{3}^{2} c_{4}\right)+16 c_{0} c_{2}^{4} c_{4} \\
& -192 c_{0}^{2} c_{1} c_{3} c_{4}^{2}-80 c_{0} c_{1} c_{2}^{2} c_{3} c_{4}
\end{aligned}
$$

[1] Some early studies on the renormalization group include E. C. G. Stueckelberg and A. Peterman, Helv. Phys. Acta 26, 499 (1953); M. Gell-Mann and F. Low, Phys. Rev. 95, 1300 (1954); N. N. Bogolubov and D. V. Shirkov, Doklad. Akad. Nauk SSSR 103, 391 (1955); C. G. Callan, Phys. Rev. D 2, 1541 (1970); K. Symanzik, Commun. Math. Phys. 18, 227 (1970); K. Wilson, Phys. Rev. D 3, 1818
(1971).

[2] Another common normalization uses $\mathcal{L}_{\text {int }}=$ $-\left(\lambda_{\text {alt }} / 4\right)\left(\vec{\phi}^{2}\right)^{2}$. These are equivalent, with $\lambda=6 \lambda_{\text {alt. }}$.

[3] See, e.g., K. G. Wilson and J. Kogut, Phys. Repts. 12, 75 (1974); M. Aizenman, Commun. Math. Phys. 82, 69 (1982); B. Freedman, P. Smolensky, and D. Weingarten, Phys. Lett. B 113, 491 (1982); J. Fröhlich, Nucl. Phys. 
B 200, 281 (1982); R. F. Dashen and H. Neuberger, Phys. Rev. Lett. 50, 1897 (1983); M. Aizenman and R. Graham, Nucl. Phys. B 225, 261 (1983); C. B. Lang, Nucl. Phys. B 240, 577 (1984); J. Kuti, L. Lin, and Y. Shen, Phys. Rev. Lett. 61, 678 (1988); M. Lüscher and P. Weisz, Nucl. Phys. B 290, 25 (1987); M. Lüscher and P. Weisz, Nucl. Phys. B 318, 705 (1989); D. J. E. Callaway, Phys. Repts. 167, 241 (1988), and references therein.

[4] S. Weinberg, The Quantum Theory of Fields (Cambridge Univ. Press, Cambridge, 1996), vol. II, ch. 18.

[5] J. Zinn-Justin, Quantum Field Theory and Critical Phenomena, 4th ed. (Oxford Univ. Press, Oxford, 2002).

[6] H. J. Schnitzer, Phys. Rev. D 10, 1800 (1974); S. R. Coleman, R. Jackiw, and H. D. Politzer, Phys. Rev. D 10, 2491 (1974); L. F. Abbott, J. S. Kang, and H. J. Schnitzer, Phys. Rev. D 13, 2212 (1976); W. A. Bardeen and M. Moshe, Phys. Rev. D 28, 1372 (1983).

[7] S. R. Coleman and E. J. Weinberg, Phys. Rev. D 7, 1888 (1973). E. Gildener and S. Weinberg, Phys. Rev. D 13, 3333 (1976); an early review is M. Sher, Phys. Repts. 179, 273 (1989).

[8] R. Shrock, Phys. Rev. D 89, $045019 \quad$ (2014) arXiv: 1311.5268 .

[9] B. Holdom, Phys. Lett. B 694, 74 (2010) arXiv:1006.2119.

[10] W. A. Bardeen, B. W. Lee, and R. E. Shrock, Phys. Rev. D 14, 985 (1976); E. Brézin and J. Zinn-Justin, Phys. Rev. B 14, 3110 (1976); see also A. Polyakov, Phys. Lett. B 59, 79 (1975).

[11] S. Weinberg, in A. Zichichi, ed., Proceedings of the International School of Subnuclear Physics, Erice (1976), p. 790.

[12] E. Brézin, J. C. Le Guillou, and J. Zinn-Justin, Phys. Rev. D 9, 1121 (1974).

[13] G. 't Hooft, M. Veltman, Nucl. Phys. B 44, 189 (1972).

[14] W. A. Bardeen, A. J. Buras, D. W. Duke, and T. Muta, Phys. Rev. D 18, 3998 (1978). See also G. 't Hooft, Nucl. Phys. B 61, 455 (1973).

[15] H. Kleinert, J. Neu, V. Schulte-Frohlinde, K. G. Chetyrkin, and S. A. Larin, Phys. Lett. B 272, 39 (1991); Erratum: Phys. Lett. B 319, 545 (1993).

[16] H. Kleinert and V. Schulte-Frohlinde, Critical Properties of $\phi^{4}$ Theories (World Scientific, Singapore, 2001).

[17] A. A. Vladimirov, D. I. Kazakov, and O. V. Tarasov, Zh. Eksp. Teor. Fiz. 77, 1035 (1979) [Sov. Phys. J.E.T.P. 50, 521 (1979)].

[18] Here and below, when expressions are given for $N$ that evaluate to non-integral real values, it is understood that they are formal and are interpreted via an analytic continuation of $N$ from physical nonnegative integer values to real numbers.

[19] L. N. Lipatov, Sov. Phys. JETP 45, 216 (1977) [Zh. Eksp. Teor. Fiz. 72, 411 (1977)]; E. Brézin, J. C. Le Guillou, and J. Zinn-Justin, Phys. Rev. D 15, 1544 (1977); G. Parisi, Phys. Lett. B 66, 167 (1977); M. C. Bergère and F. David, Phys. Lett. B 135, 412 (1984); J. C. Le Guillou and J. Zinn-Justin, eds., Large Order Behavior of Perturbation Theory (North-Holland, Amsterdam, 1990).

[20] E. Gardi and M. Karliner, Nucl. Phys. B 529, 383 (1998); E. Gardi, G. Grunberg and M. Karliner, JHEP 9807, 007 (1998).

[21] T. A. Ryttov, R. Shrock, Phys. Rev. D 83, 056011 (2011) arXiv:1011.4542.

[22] C. Pica, F. Sannino, Phys. Rev. D 83, 035013 (2011),
arXiv:1011.5917.

[23] R. Shrock, Phys. Rev. D 87, $105005 \quad$ (2013) arXiv:1301.3209.

[24] R. Shrock, Phys. Rev. D 87, 116007 (2013) arXiv:1302.5434.

[25] I. M. Gelfand, M. M. Kapranov, and A. V. Zelevinsky, Discriminants, Resultants, and Multidimensional Determinants (Birkhäuser, Boston, 1994).

[26] For a recent review, see X.-G. Wu, S. J. Brodsky, and M. Mojaza, Prog. Part. Nucl. Phys. 72, 44 (2013) arXiv:1302.0599.

[27] G. 't Hooft, in The Whys of Subnuclear Physics, Proc. 1977 Erice Summer School, ed. A. Zichichi (Plenum, New York, 1979), p. 943.

[28] T. A. Ryttov and R. Shrock, Phys. Rev. D 86, 065032 (2012) arXiv:1206.2366; T. A. Ryttov and R. Shrock, Phys. Rev. D 86, 085005 (2012) arXiv:1206.6895.

[29] R. Shrock, Phys. Rev. D 88, 036003 (2013) arXiv:1305.6524.

[30] R. Shrock, Phys. Rev. D 90, 045011 (2014) arXiv:1405.6244.

[31] A. L. Kataev and M. D. Vardiashvili, Phys. Lett. B 221, 377 (1989); Erratum Phys. Lett. B 241, 644 (1990).

[32] K. Halpern and K. Huang, Phys. Rev. D 53, 3252 (1996); T. Morris, Phys. Rev. Lett. 77, 1658 (1996); K. Halpern and K. Huang, Phys. Rev. Lett. 77, 1659 (1996); C. Bagnuls and C. Bervillier, Phys. Repts. 348, 91 (2001); F. Cooper, J. F. Dawson, and B. Mihaila, Phys. Rev. D 70, 105008 (2004); Phys. Rev. D 71, 096003 (2005); E. Caliceti, M. Meyer-Hermann, R. Ribeca, A. Surzhykov, and U. D. Jentschura, Phys. Repts. 446, 1 (2007); I. M. Suslov, Zh. Eksp. Teor. Fiz. 127, 1350 (2005) [JETP 100, 1188 (2005)]; O. J. Rosten, JHEP 07, 019 (2009); V. Rochev, J. Phys. A 44, 305403 (2011); J. Braun, H. Gies, and D. D. Scherer, Phys. Rev. D 83, 085012 (2011).

[33] G. A. Baker, Essentials of Padé Approximants (Academic Press, New York, 1975).

[34] This is different from the situation in statistical mechanics, where dlog Padé approximants have been used extensively in the analysis of series expansions for the study of critical phenomena. In the statistical mechanics application, the high-temperature series expansions and, for discrete spin models, also the low-temperature series expansions, are Taylor series with finite radii of convergence. See, e.g., 33] and I-H. Lee and R. E. Shrock, Phys. Rev. B 36, 3712 (1987) for further references.

[35] W. E. Caswell, Phys. Rev. Lett. 33, 244 (1974); D. R. T. Jones, Nucl. Phys. B 75, 531 (1974); T. Banks and A. Zaks, Nucl. Phys. B 196, 189 (1982).

[36] E. Mølgaard and R. Shrock, Phys. Rev. D 89, 105007 (2014) arXiv:1403.3058 and references therein.

[37] Some early lattice studies of scalar-fermion theories include I-H. Lee and R. E. Shrock, Phys. Rev. Lett. 59, 14 (1987); I-H. Lee, J. Shigemitsu, and R. E. Shrock, Nucl. Phys. B 330, 225 (1990); Nucl. Phys. B 334, 265 (1990). A. Hasenfratz, W.-Q. Liu, and T. Neuhaus, Phys. Lett. B 236, 339 (1990); J. Shigemitsu, Nucl. Phys. Proc. Suppl. 20, 515 (1991); R. E. Shrock, in Quantum Fields on the Computer, ed. M. Creutz (World Scientific, Singapore, 1992), pp. 150-210. Recent studies include Z. Fodor, K. Holland, J. Kuti, D. Nogradi, and C. Schroeder, Proc. Sci. LAT2007 (2007) 056 arXiv:0710.3151; P. Gerhold and K. Jansen, JHEP 1004, 094 (2010) 094 arXiv:1002.4336. 
[38] T. P. Cheng, E. Eichten, and L.-F. Li, Phys. Rev. D 9, 2259 (1974); L. Maiani, G. Parisi and R. Petronzio, Nucl. Phys. B 136, 115 (1978); M. Fischler and J. Oliensis, Phys. Rev. D 28, 2027 (1983); R. Flores and M. Sher, Phys. Rev. D 27, 1679 (1983); M. A. Bég, C. Panagiotakopoulos and A. Sirlin, Phys. Rev. Lett. 52, 883 (1984); M. E. Machacek and M. T. Vaughn, Nucl. Phys. B 236, 221 (1984); Nucl. Phys. B 249, 70 (1985); I. Jack and H. Osborn, Nucl. Phys. B 249, 472 (1985); M. Lindner, Z. Phys. C 31, 298 (1986); C. Ford, I. Jack, and D. R. T. Jones, Nucl. Phys. B 387, 373 (1992); V. Barger, M. S. Berger, and P. Ohmann, Phys. Rev. D 47, 1093 (1993); M.-x. Luo, H.-w. Wang and Y. Xiao, Phys. Rev. D 67, 065019 (2003).

[39] Some recent studies include B. Grinstein and P. Uttayarat, JHEP 1107, 038 (2011) arXiv:1105.2370; O. Antipin, S. Di Chiara, M. Mojaza, E. Mølgaard, and F. Sannino, Phys. Rev. D 86, 085009 (2012) arXiv:1205.6157. O. Antipin, M. Mojaza and F. Sannino, Phys. Lett. B 712, 119 (2012) arXiv:1107.2932; O. Antipin, M. Gillioz, E. Mølgaard, and F. Sannino, Phys. Rev. D 87, 125017 (2013) arXiv:1303.1525] D. F. Litim and F. Sannino, arXiv:1406.2337 and references therein.

[40] G. Degrassi, S. Di Vita, J. Elias-Miró, J. R. Espinosa, G. F. Giudice, G. Isidori, and A. Strumia, JHEP 08, 098 (2012) arXiv:1205.6497] and references therein.

[41] J. V. Uspensky, Theory of Equations (McGraw-Hill, New York, 1948);
TABLE I: Values of the $\bar{b}_{n}=b_{n} /\left(16 \pi^{2}\right)^{n}$, where $b_{n}$ are the $n$-loop beta function coefficients in Eqs. (2.1) and (2.3), for $1 \leq n \leq 5$, as functions of $N$ for $1 \leq N \leq 20$ and selected larger values of $N$. The notation $r$ en means $r \times 10^{n}$.

\begin{tabular}{|c|c|c|c|c|c|}
\hline \hline$N$ & $\bar{b}_{1}$ & $\bar{b}_{2}$ & $\bar{b}_{3}$ & $\bar{b}_{4}$ & $\bar{b}_{5}$ \\
\hline 1 & 0.2387 & -0.03588 & 0.01640 & -0.01089 & 0.09090 \\
2 & 0.2653 & -0.04222 & 0.02013 & -0.01406 & 0.01227 \\
3 & 0.2918 & -0.04855 & 0.02401 & -0.01755 & 0.01595 \\
4 & 0.3183 & -0.05488 & 0.02805 & -0.02137 & 0.02016 \\
5 & 0.3448 & -0.06121 & 0.03224 & -0.02553 & 0.02492 \\
6 & 0.3714 & -0.06755 & 0.03658 & -0.03001 & 0.03024 \\
7 & 0.3979 & -0.07388 & 0.04108 & -0.03482 & 0.03616 \\
8 & 0.4244 & -0.08021 & 0.04573 & -0.03996 & 0.04269 \\
9 & 0.4509 & -0.08655 & 0.05054 & -0.04542 & 0.04984 \\
10 & 0.4775 & -0.09288 & 0.05550 & -0.05121 & 0.05765 \\
100 & 2.8648 & -0.6628 & 1.1324 & -1.87505 & 5.4152 \\
200 & 5.5174 & -1.2961 & 3.7918 & -6.7359 & 26.0096 \\
300 & 8.1700 & -1.9293 & 7.9910 & -14.2812 & 54.2973 \\
400 & 10.8225 & -2.5626 & 13.7300 & -24.2014 & 63.0752 \\
500 & 13.4751 & -3.1958 & 21.0087 & -36.1873 & 5.4300 \\
600 & 16.1277 & -3.8291 & 29.8273 & -49.9293 & $-1.85262 \mathrm{e} 2$ \\
700 & 18.7803 & -4.4624 & 40.1856 & -65.1180 & $-5.95335 \mathrm{e} 2$ \\
800 & 21.4329 & -5.0956 & 52.0837 & -81.4440 & $-1.33083 \mathrm{e} 3$ \\
$1.0 \mathrm{e} 3$ & 26.7380 & -6.3621 & 80.4992 & $-1.16270 \mathrm{e} 2$ & $-4.30084 \mathrm{e} 3$ \\
$2.0 \mathrm{e} 3$ & 53.2639 & -12.6947 & $3.149645 \mathrm{e} 2$ & $-2.53435 \mathrm{e} 2$ & $-1.01045 \mathrm{e} 5$ \\
$3.0 \mathrm{e} 3$ & 79.7897 & -19.0273 & $7.03408 \mathrm{e} 2$ & $-1.02078 \mathrm{e} 2$ & $-5.63816 \mathrm{e} 5$ \\
$4.0 \mathrm{e} 3$ & $1.063155 \mathrm{e} 2$ & -25.3598 & $1.24583 \mathrm{e} 3$ & $6.472275 \mathrm{e} 2$ & $-1.86330 \mathrm{e} 6$ \\
\hline \hline
\end{tabular}




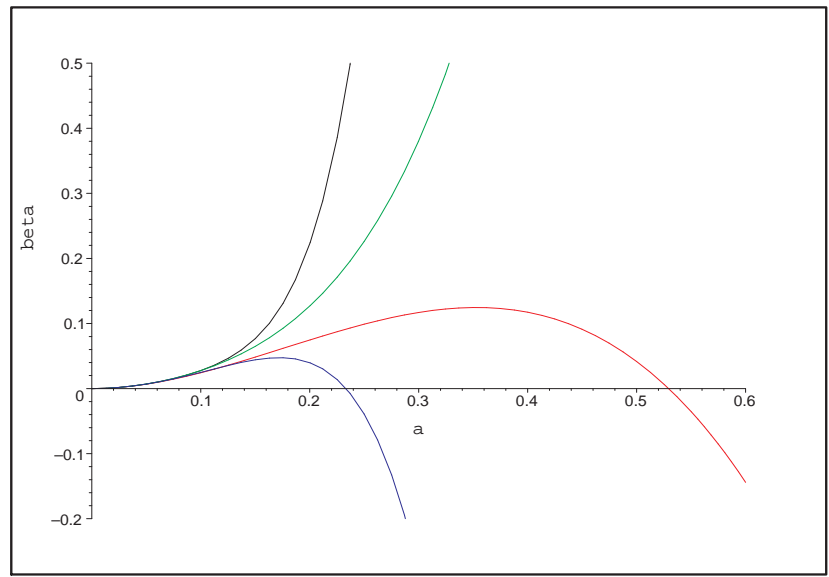

FIG. 1: Plot of the $n$-loop $\beta$ function $\beta_{a, n \ell}$ as functions of $a$ for $N=1$ and (i) $n=2$ (red), (ii) $n=3$ (green), (iii) $n=4$ (blue), and $n=5$ (black) (colors in online version). At $a=0.18$, going from bottom to top, the curves are for $n=4, n=2, n=3$, and $n=5$.

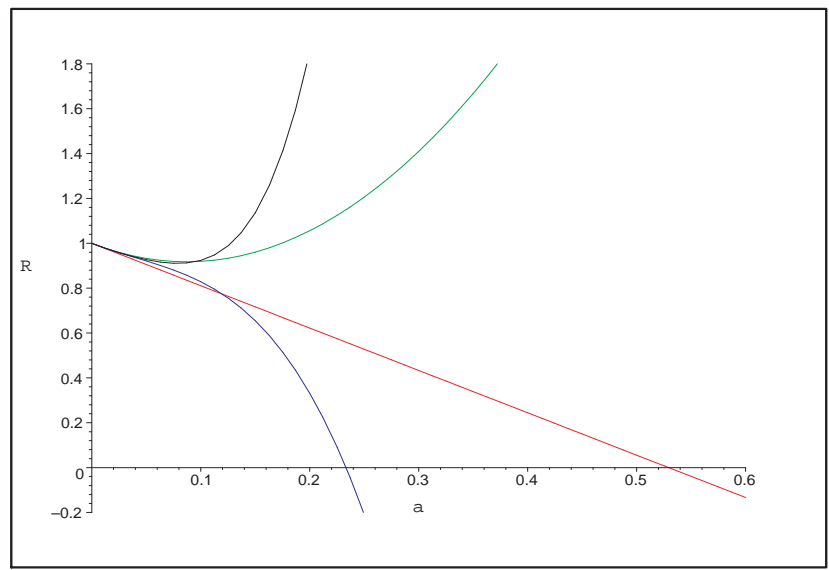

FIG. 2: Plot of the ratio $R \equiv R_{a, n}$ of $\beta_{a, n \ell}$ divided by $\beta_{a, 1 \ell}$, as a function of $a$ for $N=1$ and (i) $n=2$ (red), (ii) $n=3$ (green), (iii) $n=4$ (blue), and $n=5$ (black) (colors in online version). At $a=0.18$, going from bottom to top, the curves are for $n=4$, $n=2, n=3$, and $n=5$. 


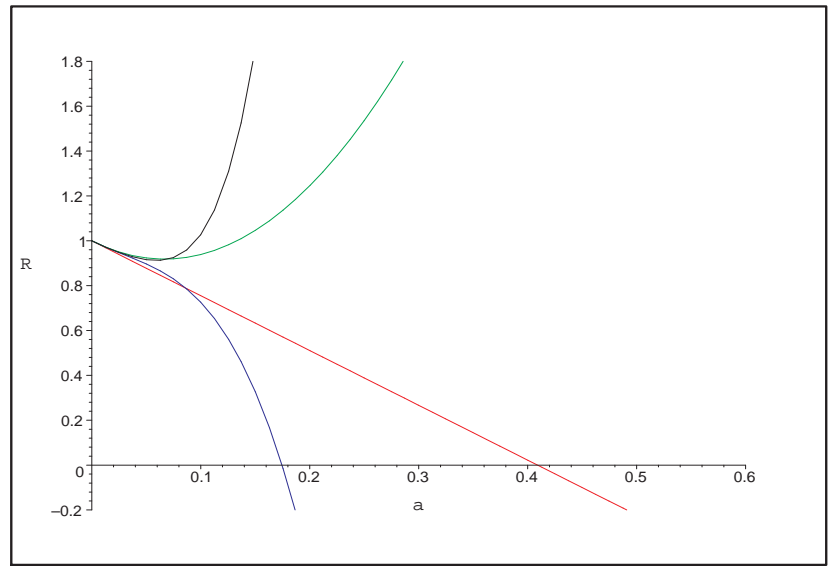

FIG. 3: Plot of the ratio $R \equiv R_{a, n}$ of $\beta_{a, n \ell}$ divided by $\beta_{a, 1 \ell}$, as a function of $a$ for $N=10$ and (i) $n=2$ (red), (ii) $n=3$ (green), (iii) $n=4$ (blue), and $n=5$ (black) (colors in online version). At $a=0.14$, going from bottom to top, the curves are for $n=4$, $n=2, n=3$, and $n=5$.

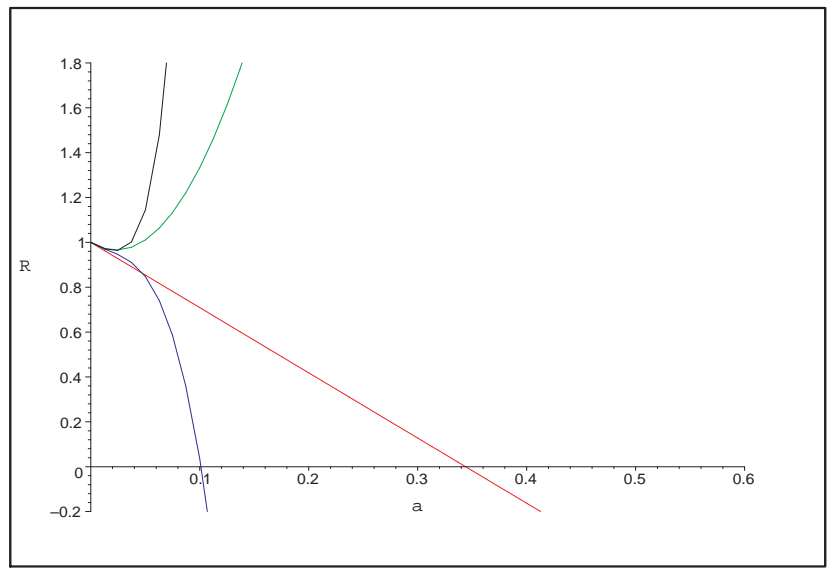

FIG. 4: Plot of the ratio $R \equiv R_{a, n}$ of $\beta_{a, n \ell}$ divided by $\beta_{a, 1 \ell}$, as a function of $a$ for $N=100$ and (i) $n=2$ (red), (ii) $n=3$ (green), (iii) $n=4$ (blue), and $n=5$ (black) (colors in online version). At $a=0.06$, going from bottom to top, the curves are for $n=4$, $n=2, n=3$, and $n=5$. 
TABLE II: Values of the UV zero $a_{U V, n \ell}$ of the $n$-loop beta function, $\beta_{a, n \ell}$, for $n=2, \ldots, 5$, as a function of $N$, with $b_{n}, n=3,4,5$ calculated in the $\overline{\mathrm{MS}}$ scheme. The dash notation - means that $\beta_{a^{\prime}, n \ell}$ has no physical UV zero. The special values of $N$ are $N_{\Delta_{4}}=493.096$ from Eq. (3.10), $N_{b 5 z}=504.740$ from Eq. 2.15), and $N_{b 4 z}=3218.755$ from Eq. 2.12).

\begin{tabular}{|c|c|c|c|c|}
\hline \hline$N$ & $a_{U V, 2 \ell}$ & $a_{U V, 3 \ell}$ & $a_{U V, 4 \ell}$ & $a_{U V, 5 \ell}$ \\
\hline 1 & 0.5294 & - & 0.2333 & - \\
2 & 0.5000 & - & 0.2217 & - \\
3 & 0.4783 & - & 0.2123 & - \\
4 & 0.4615 & - & 0.2044 & - \\
5 & 0.4483 & - & 0.1978 & - \\
6 & 0.4375 & - & 0.1920 & - \\
7 & 0.4286 & - & 0.1869 & - \\
8 & 0.42105 & - & 0.1823 & - \\
9 & 0.4146 & - & 0.1783 & - \\
10 & 0.4091 & - & 0.1746 & - \\
100 & 0.3439 & - & 0.1012 & - \\
$N_{\Delta_{4}} z$ & 0.3356 & - & 0.07353 & 0.12636 \\
500 & 0.3355 & - & 0.07341 & 0.08045 \\
$N_{b 5 z}$ & 0.3355 & - & 0.073325 & 0.073325 \\
1000 & 0.3344 & - & 0.07241 & 0.02276 \\
2000 & 0.3339 & - & 0.1054 & 0.01231 \\
3000 & 0.3337 & - & 0.5475 & 0.008850 \\
$N_{b 4 z}$ & 0.3337 & - & - & 0.008366 \\
4000 & 0.3336 & - & - & 0.007042 \\
$10^{4}$ & 0.3334 & - & - & 0.003460 \\
\hline \hline
\end{tabular}

TABLE III: Values of the transformation function $f\left(a_{U V, 2 \ell}^{\prime}\right)$ and resultant UV zeros $a_{U V, n \ell}^{\prime}$ of the $n$-loop beta function, $\beta_{a^{\prime}, n \ell}$, for $n=2, \ldots, 5$, as a function of $N$, resulting from the application of the $S_{R, 2}$ scheme transformation to the $\overline{\mathrm{MS}}$ scheme. The dash notation - means that $\beta_{a, n \ell}$ has no physical UV zero.

\begin{tabular}{|c|c|c|c|c|}
\hline \hline$N$ & $f\left(a_{U V, 2 \ell}^{\prime}\right)$ & $a_{U V, 2 \ell}^{\prime}, n=2,3$ & $a_{U V, 4 \ell}^{\prime}$ & $a_{U V, 5 \ell}^{\prime}$ \\
\hline 1 & 4.0410 & 0.5294 & 0.1917 & - \\
2 & 3.9961 & 0.5000 & 0.1822 & - \\
3 & 3.9726 & 0.4783 & 0.1746 & - \\
4 & 3.9642 & 0.4615 & 0.1683 & - \\
5 & 3.9668 & 0.4483 & 0.1630 & - \\
6 & 3.9776 & 0.4375 & 0.1584 & - \\
7 & 3.9947 & 0.4286 & 0.1544 & - \\
8 & 4.0167 & 0.42105 & 0.1509 & - \\
9 & 4.0427 & 0.4146 & 0.1477 & - \\
10 & 4.0719 & 0.4091 & 0.1448 & - \\
100 & 8.3846 & 0.3439 & 0.08354 & - \\
\hline \hline
\end{tabular}

